\title{
Alternative splicing generates novel Fads3 transcript in mice
}

\author{
Ji Yao Zhang" ${ }^{\# 1}$ Xia Qin ${ }^{\# 1,2}$, Hui Gyu Park${ }^{1}$, Ellen Kim¹, Guowen Liu², Kumar S. D. \\ Kothapalli ${ }^{1}$, and J. Thomas Brenna ${ }^{1}$ \\ ${ }^{1}$ Division of Nutritional Sciences, Cornell University, Ithaca, NY 14853, USA \\ 2 College of Veterinary Medicine, Jilin University, Changchun, Jilin, China \\ \# These authors contributed equally to this work.
}

\begin{abstract}
Fads 3 is the third member of the fatty acid desaturase gene cluster; with at least eight evolutionarily conserved alternative transcripts (AT), having no clearly established function as are known for FADS2 and FADS1. Here we present identification of a novel Fads 3 transcript in mice (Fads3AT9), characterize Fads3AT9 expression in mouse tissues and evaluate correlations with metabolite profiles. Total RNA obtained from mouse tissues is reverse-transcribed into cDNA and used as template for PCR reactions. Tissue fatty acids were extracted and quantified by gas chromatography. Sequencing analysis revealed complete absence of exon 2 resulting in an open reading frame of $1239 \mathrm{bp}$, encoding a putative protein of 412 aa with loss of 37 aa compared to classical Fads3 (Fads3CS). FADS3AT9 retains all the conserved regions characteristic of front end desaturase (cytochrome b5 domain and three histidine repeats). Both Fads3CS and Fads3AT9 are ubiquitously expressed in 11 mouse tissues. Fads $3 A T 9$ abundance was greater than Fads $3 C S$ in pancreas, liver, spleen, brown adipose tissue and thymus. Fads $3 C S$ expression is low in pancreas while Fads3AT9 is over ten-fold greater abundance. The eicosanoid precursor fatty acid 20:4n - 6, the immediate desaturation product of the Fads 1 coded $\Delta 5$-desaturase, was highest in pancreas where Fads $3 C S$ is low. Changes in expression patterns and fatty acid profiles suggest that Fads3AT9 may play a role in the regulation and/or biosynthesis of long chain polyunsaturated fatty acids from precursors.
\end{abstract}

\section{Keywords}

Fatty acid desaturase; Alternative transcript; Alternative splicing; Long chain polyunsaturated fatty acids

\footnotetext{
Kumar S. D. Kothapalli ksk25@cornell.edu, J. Thomas Brenna jtb4@cornell.edu.

Electronic supplementary material The online version of this article (doi:10.1007/s11033-016-4018-7) contains supplementary material, which is available to authorized users.

Author Contributions J.T.B., K.S.D.K., X.Q., J.Y.Z. designed research; X.Q., J.Y.Z., H.P., and E.K. executed the research; J.T. B., K.S.D.K., and G.L. contributed new reagents/analytic tools; J.T.B., K.S.D.K., X.Q., and J.Y.Z. analyzed and interpreted the data; and J.T. B., K.S.D.K., X.Q., and J.Y.Z. wrote the first draft and all authors approved the final draft.

Compliance with ethical standards

Conflict of interest All authors declare no conflict of interest.
} 


\section{Introduction}

Polyunsaturated fatty acids (PUFA) are ubiquitous in mammalian tissue. Long chain PUFA (LCPUFA) are bioactive components of membrane phospholipids and serve as substrates for signaling molecules [1]. They can be obtained from the diet or are endogenously synthesized in the liver from dietary PUFA precursors, alpha-linolenic acid (ALA, 18:3n - 3) and linoleic acid (LA, 18:2n - 6) by alternating series of position-specific desaturation and carbon chainelongation reactions [2]. The nonheme, iron-containing, oxygen-dependent fatty acid desaturases catalyze introduction of double bonds at specific positions within a fatty acid chain. FADS1 (OMIM\#606148), FADS2 (OMIM\#606149) and FADS3 (OMIM\#606150) are located as a cluster within $100 \mathrm{~kb}$ region on the long arm of HSA11q12-13.1 locus, while a similar organization is found on mouse chromosome 19 [3, 4]. All three fatty acid desaturase (FADS) genes have evolved by gene duplication events, share 12 exons and 11 introns, and contain a well conserved cytochrome b5 domain and three histidine repeats [4].

The FADS1 and FADS2 genes encode classical transcripts (CS) for the $\Delta 5$-desaturase and $\Delta 4-/ \Delta 6$-/ $\Delta 8$-desaturase, respectively [2, 5]. Direct evidence for the biochemical function of FADS3 gene product is enigmatic. The first Fads3 null mouse is fertile despite its high expression at the implantation site in the mouse uterus [6] and has no overt phenotype, though the biochemical phenotype suggests a role in docosahexaenoic acid (DHA, 22:6n - 3) synthesis [7]. An early in vitro report suggests a unique role as a back end desaturase for trans-11 vaccenic acid [8]. Genetic studies have shown associations with familial combined hyperlipidemia in a Mexican population [9] and a minor allele of FADS3 SNP (rs174455) was found to be negatively associated with DHA in red blood cell phospholipids in the Alspac cohort [10].

We established that primate FADS3 gives rise to at least eight alternative transcripts that are highly expressed in a tissue-specific manner and are phylogenetically conserved [11]. Others have reported detection of FADS3 proteins [12]. We have shown with mouse embryonic fibroblast (MEF) cells and ribosome foot-printing technology the first positive-sequencespecific-proof of FADS3 translation [5]. Additionally, both FADS1 and FADS2 are also alternatively spliced, and we have established a function for a FADS1 AT in FADS2 mediated desaturation $[5,13]$. In this study, we report a transcript variant of Fads3 in adult mouse liver generated by alternative splicing, evidence of its wide expression in 11 mouse tissues. We also report fatty acid profiles emphasizing the FADS target metabolites from 11 different tissues from $n=4$ mice.

\section{Materials and methods}

Animals

Studies on mice were approved by Cornell University Institutional Animal Care and Use Committee (IACUC, protocol \# 2011-0007). C57BL/6 mice were maintained on rodent laboratory diet (7012 Teklad LM-485, Harlan Laboratories, WI) and were fed ad libitum. High quality adult mouse tissues, treated with RNAlater, and maintained at $-80^{\circ} \mathrm{C}$, were used to isolate total RNA. We used four mice ( 2 males and 2 females) average 1 year old at the time of sacrifice. 


\section{RNA isolation and cDNA synthesis}

Total RNA was isolated from adult mouse tissues using the E.Z.N.A. Total RNA kit (Omega Bio-Tek, GA). The quality and quantity of RNA was analyzed by $260 / 280 \mathrm{~nm}$ ratios using a low volume spectrophotometer (NanoDrop, Thermo Scientific, DE). Total RNA was reverse transcribed into cDNA using the High Capacity cDNA Reverse Transcription Kit (Life Technologies, NY) according to the manufacturer's instructions.

\section{RT-PCR conditions and identification of alternative transcript}

We designed primers to amplify the protein coding region of mouse Fads3 (GenBank Accession\# NM_021890). Fads3 Forward: ATGGGCGGTGTCGGGGAGCCCGGA and Fads3 Reverse: TCATTGATGGAGGTATGCATCCAGCCA. RT-PCR was performed using these primers with adult female mouse liver cDNA as template and iProof high-fidelity DNA polymerase (BIO_RAD, CA) in a $20 \mu \mathrm{l}$ reaction. Cycling conditions were: initial denaturation at $98{ }^{\circ} \mathrm{C}$ for $30 \mathrm{~s}$ followed by 30 cycles of denaturation at $98{ }^{\circ} \mathrm{C}$ for $10 \mathrm{~s}$, annealing at $68{ }^{\circ} \mathrm{C}$ for $30 \mathrm{~s}$ and extension at $72{ }^{\circ} \mathrm{C}$ for $30 \mathrm{~s}$, with a final extension at $72{ }^{\circ} \mathrm{C}$ for $5 \mathrm{~min}$. PCR products were run on $2 \%$ agarose gels containing ethidium bromide and visualized under UV. Two prominent bands were obtained when separated by electrophoresis on a $2 \%$ agarose gel. The lower band was gel purified, cloned into p GEM T-Easy vector (Promega, WI) and sequenced using T7 forward and SP6 reverse universal primers at the Cornell University life sciences core laboratories.

\section{Expression of Fads $3 C S$ and $A T 9$ in mouse tissues}

To amplify Fads3CS, a forward primer was designed within the exon 1 and reverse primer was designed within in the exon 2 which is missing in the alternative transcript. To amplify Fads3AT9, the forward primer bridged the deleted parts of the exon 2. Details of the primers and PCR conditions are provided in Supplementary Table 1. Briefly, cDNA was prepared from 11 different mouse tissues using the High Capacity cDNA Reverse Transcription Kit (Life Technologies, NY) to measure mRNA expression levels of Fads3CS and Fads3AT9. PCR amplification reactions were performed using EmeraldAmp GT PCR Master Mix (Clontech, CA) in a volume of 20 microliters. PCR products were resolved using $2 \%$ agarose gels and bands visualized under UV light. $18 S$ was used as control. mRNA expression of Fads $3 C S$ and $A T 9$ were quantified using ImageJ software (National Institutes of Health, USA) and the expression levels were normalized to reference gene 18S. mRNA expression of Fads3CS was taken as "1" and the changes between $C S$ and $A T 9$ were measured by using ratio of AT9/CS.

\section{Fatty acid analysis}

Adult mouse tissues (11 different tissues from 4 mice) were used for fatty acid extraction and analysis. Fatty acid methyl esters (FAME) were prepared using modified one-step method of Garces and Mancha [14] modified for use with soft tissue. Methylated fatty acids were quantified by gas chromatography-flame ionization detection (GC-FID) using an equal weight mixture for response factor calibration and peak structures were identified by GCcovalent adduct chemical ionization tandem mass spectrometry (GC-CACI-MS/MS) [2]. 
Three injections were performed for each FAME and quantity of each fatty acid species was calculated as percentage by weight in total fatty acids $(\%, \mathrm{w} / \mathrm{w})$.

\section{Statistical analysis}

Data are expressed as mean \pm SD. Statistical significance of fatty acids among eleven tissues was analyzed using one-way ANOVA and Tukey's post hoc test (SPSS software, version 16.0). Different alphabets shown in Table 1 represent statistical significance $(P<0.05)$ between groups. Paired Student's $t$ Test was used to analyze the changes in gene expression levels of Fads3AT9 and Fads $3 C S$ within each tissue $(* P<0.05)$.

\section{Results and discussion}

The open reading frame sequence of house mouse (Mus musculus) Fads3 (GenBank Accession\# NM_021890) consists of 1350 bp, encoding a protein of 449 aa and a stop codon. To clone Fads3 ORF into expression vectors for functional studies we designed primers to amplify $1350 \mathrm{bp}$ of Fads 3 , however, in addition to the expected product size we observed another prominent band using adult mouse liver cDNA (Fig. 1, Top). We carefully gel extracted the prominent band, cloned and sequenced the product resulting in the identification of a novel splice variant of Fads3 (Fads3AT9; GenBank Accession\# KM975938). Sequencing analysis revealed complete absence of exon 2 (Fig. 1, Bottom). The putative 1239 bp ORF of Fads3AT9 identified using ORF finder <http:// www.ncbi.nlm.nih.gov/gorf/gorf.html>, encodes a protein of 412 aa, resulting from an inframe loss of 37 aa compared to classical FADS3. FADS3AT9 retains all the conserved regions characteristic of front end desaturase (cytochrome b5 domain and three histidine repeats) (Supplement Fig. 1).

We studied the expression of Fads3AT9 along with Fads3CS using 11 mouse tissues. AT9 along with $C S$ is ubiquitously expressed in all 11 tissues tested; $A T 9$ expression is higher than $C S$ in pancreas, liver, spleen, brown adipose tissue and thymus (Fig. 2, supplementary Fig. 2). $C S$ expression is low in pancreas while $A T 9$ is at much greater abundance (Fig. 2, supplementary Fig. 2). These observations suggest that $A T 9$ has an important role in pancreatic LCPUFA synthesis.

We used Cell-PLoc 2.0 <http://www.csbio.sjtu.edu.cn/bioinf/euk-multi-2/>[15] to predict subcellular localization of Fads3CS and Fads3AT9. The protein sequences of Fads3CS and Fads3AT9 in Fasta format were uploaded and the software predicted putative subcellular localization. Both Fads $3 C S$ and Fads $3 A T 9$ are predicted to be localized to endoplasmic reticulum (ER) with high confidence. Fads proteins are known to localize to the ER though recently organelle-specific staining and Western blotting show that primate FADS1 and FADS2 localize to both ER and mitochondria [2,5]. By using the protein molecular weight calculator <http://www.sciencegateway.org/tools/proteinmw.htm>, we predict protein size of $51 \mathrm{kDa}$ for FADS3CS and $47 \mathrm{kDa}$ for FADS3AT9. Earlier, Pedrono et al. [12] also reported existence of several FADS3 protein isoforms and Shoji et al. reported two FADS2 isoforms with sizes of 52 and $46 \mathrm{kDa}$, respectively [16]. 
We also checked the relevant substrate-product metabolites via fatty acid profiles of the 11 tissues (Table 1). Table 1 is arranged based on highest to lowest total $n-6$ PUFA levels. Total $n$ - 6 PUFA levels were highest in the pancreas, followed by liver and spleen and total $\mathrm{n}$ - 3 PUFA was highest in cortex and hippocampus, followed by cerebellum. Among these tissues, docosahexaenoic acid (22:6n - 3) was highest in the cortex and hippocampus. We found that the twenty carbon eicosanoid precursor arachidonic acid (20:4n - 6), the immediate product of the FADS1 $\triangle 5$-desaturase, was highest in pancreas and spleen (Table 1). Earlier, it has been shown that $20: 4 n-6$ is significantly higher in the pancreatic phospholipid fraction compared to liver and adipose [17]. AT9 expression was high but Fads3CS low in pancreas, suggesting that AT9 may be playing a key role in LCPUFA biosynthesis in this tissue via direct catalysis by an AT9 protein, or some modification of the Fads1CS catalytic function. Alternative splicing regulates enzymatic activities of the proteins, their interactions with ligands and substrate specificity [18]. We previously reported that a $F A D S 1$ splice variant (FADS1AT1) potentiates $F A D S 2$ mediated production of 18:3n - 6 [5]. Widespread expression patterns and fatty acid profiles in mouse tissues suggest that Fads $3 A T 9$ may play an important role in the regulation and/or biosynthesis of LCPUFA from precursors.

\section{Supplementary Material}

Refer to Web version on PubMed Central for supplementary material.

\section{Acknowledgments}

This work was supported by NIH grant R01 AT007003 from the National Center for Complementary and Integrative Health (NCCIH) and the Office of Dietary Supplements (ODS). Its contents are solely the responsibility of the authors and do not necessarily represent the official views of the NIH.

\section{References}

1. Park WJ, et al. FADS2 function loss at the cancer hotspot 11q13 locus diverts lipid signaling precursor synthesis to unusual eicosanoid fatty acids. Plos One. 2011; 6(11):e28186. [PubMed: 22140540]

2. Park HG, et al. The fatty acid desaturase 2 (FADS2) gene product catalyzes Delta4 desaturation to yield n-3 docosahexaenoic acid and n-6 docosapentaenoic acid in human cells. FASEB J. 2015; 29(9):3911-3919. [PubMed: 26065859]

3. Marquardt A, et al. cDNA cloning, genomic structure, and chromosomal localization of three members of the human fatty acid desaturase family. Genomics. 2000; 66(2):175-183. [PubMed: 10860662]

4. Nakamura MT, Nara TY. Structure, function, and dietary regulation of delta6, delta5, and delta9 desaturases. Annu Rev Nutr. 2004; 24:345-376. [PubMed: 15189125]

5. Park WJ, et al. A novel FADS1 isoform potentiates FADS2-mediated production of eicosanoid precursor fatty acids. J Lipid Res. 2012; 53(8):1502-1512. [PubMed: 22619218]

6. $\mathrm{Ma} \mathrm{XH}$, et al. Serial analysis of gene expression in mouse uterus at the implantation site. J Biol Chem. 2006; 281(14):9351-9360. [PubMed: 16434403]

7. Zhang J, et al. Fatty acid desaturase 3 (Fads3) null mouse biochemical phenotype. FASEB J. 2014; 28(1 Supplement):246.

8. Rioux V, et al. Trans-vaccenate is Delta13-desaturated by FADS3 in rodents. J Lipid Res. 2013; 54(12):3438-3452. [PubMed: 24070791] 
9. Plaisier CL, et al. A systems genetics approach implicates USF1, FADS3, and other causal candidate genes for familial combined hyperlipidemia. PLoS Genet. 2009; 5(9):e1000642. [PubMed: 19750004]

10. Koletzko B, et al. Genetic variants of the fatty acid desaturase gene cluster predict amounts of red blood cell docosahexaenoic and other polyunsaturated fatty acids in pregnant women: findings from the avon longitudinal study of parents and children. Am J Clin Nutr. 2011; 93(1):211-219. [PubMed: 21106917]

11. Park WJ, et al. Novel fatty acid desaturase 3 (FADS3) transcripts generated by alternative splicing. Gene. 2009; 446(1):28-34. [PubMed: 19573581]

12. Pedrono F, et al. The fatty acid desaturase 3 gene encodes for different FADS3 protein isoforms in mammalian tissues. J Lipid Res. 2010; 51(3):472-479. [PubMed: 19752397]

13. Park WJ, et al. Alternative splicing generates a novel FADS 2 alternative transcript in baboons. Mol Biol Rep. 2010; 37(5):2403-2406. [PubMed: 19693691]

14. Garces R, Mancha M. One-step lipid extraction and fatty acid methyl esters preparation from fresh plant tissues. Anal Biochem. 1993; 211(1):139-143. [PubMed: 8323025]

15. Chou KC, Shen HB. Cell-PLoc: a package of Web servers for predicting subcellular localization of proteins in various organisms. Nat Protoc. 2008; 3(2):153-162. [PubMed: 18274516]

16. Shoji Y, et al. Preparation of antiserum against rat delta6-desaturase and its use to evaluate the desaturase protein levels in rats treated with gemfibrozil, a ligand for peroxisome proliferatoractivated receptor alpha. Biosci Biotechnol Biochem. 2003; 67(5):1177-1178. [PubMed: 12834305]

17. Pinnick KE, et al. Pancreatic ectopic fat is characterized by adipocyte infiltration and altered lipid composition. Obesity (Silver Spring). 2008; 16(3):522-530. [PubMed: 18239594]

18. Kelemen O, et al. Function of alternative splicing. Gene. 2013; 514(1):1-30. [PubMed: 22909801] 
12
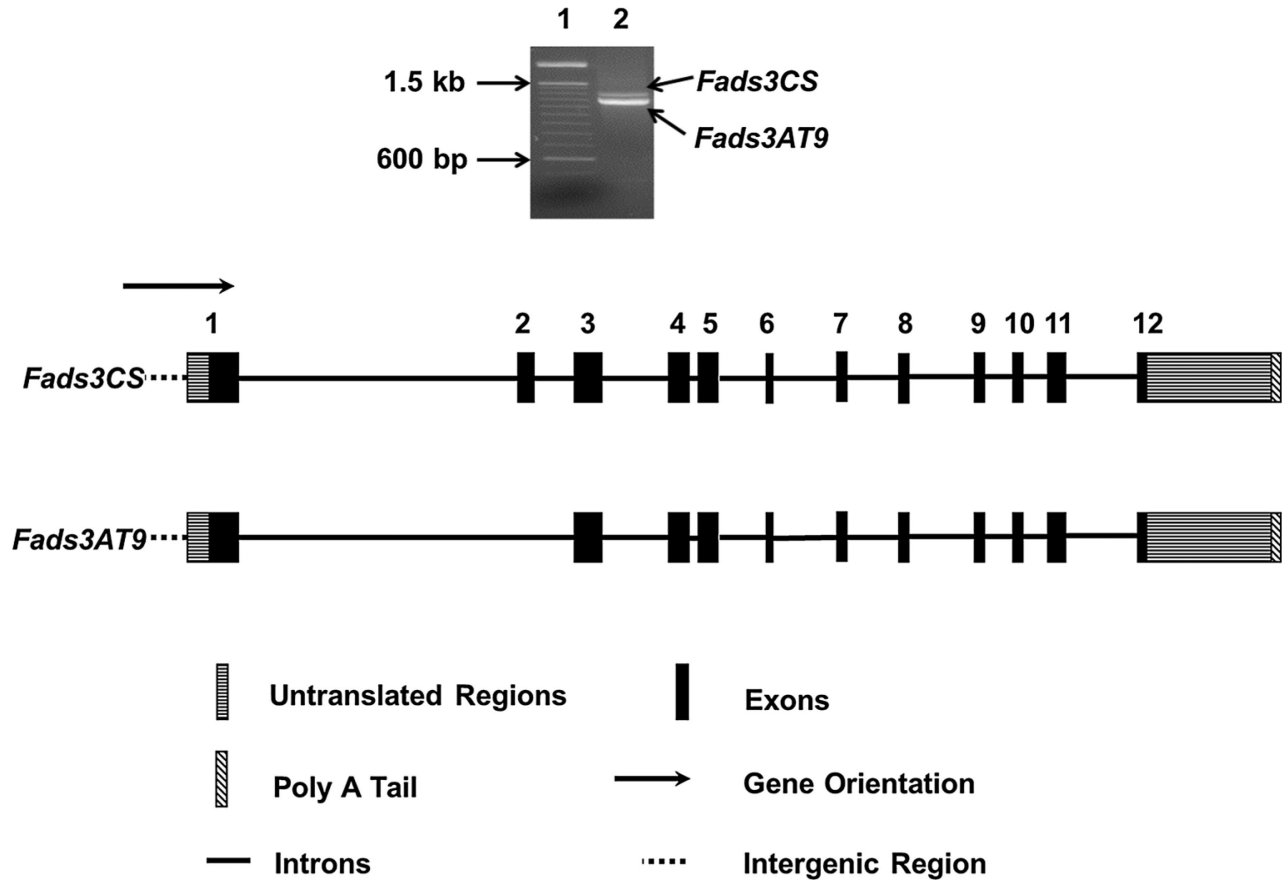

Fig. 1.

Amplicons of Fads3CS and Fads3AT9 and cartoon image of Fads3CS and Fads3AT9 gene structure Top: Fads3CS-Classical splicing, Fads3AT9-alternative transcript. The products were separated on $2 \%$ agarose gel and visualized under UV using ethidium bromide. Lane 1: 100 base pair molecular weight marker and Lane 2: PCR products amplified by RT-PCR. Bottom: Fads3CS-Classical splicing, Fads3AT9 with missing exon 2 is shown. Numbers 112 are exons 


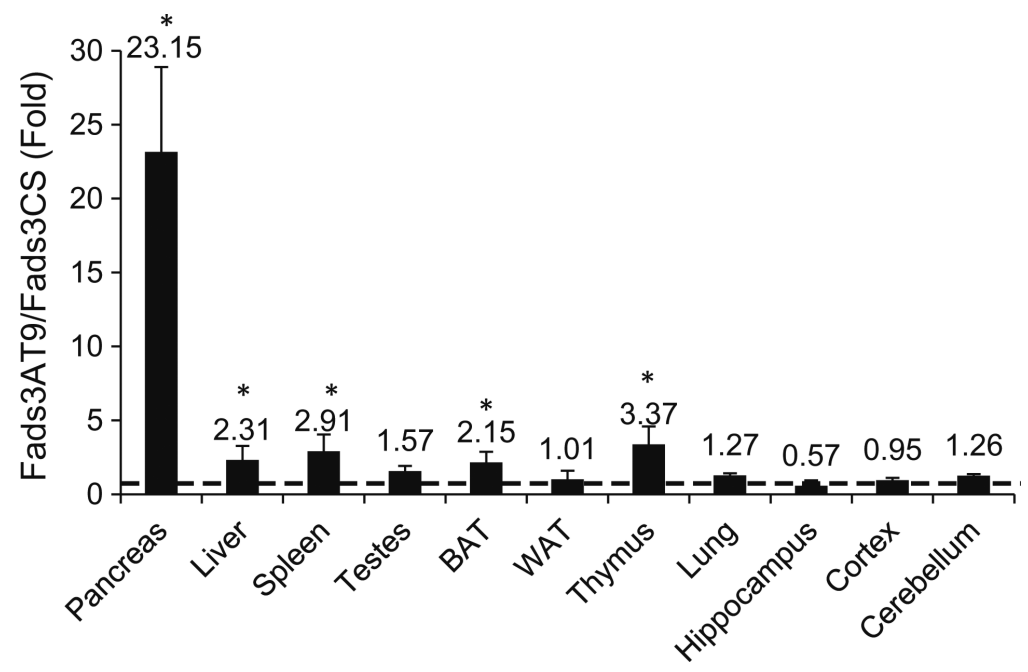

Fig. 2.

Determination of mRNA expression of Fads3AT9 in eleven tissues using Image $\mathrm{J}$ software. Relative mRNA levels of Fads3CS and Fads3AT9 were normalized to reference gene $18 S$. mRNA expression of Fads3CS was regarded as "1" (dash line) and the change between $C S$ and $A T 9$ was measured by ratio of $A T 9 / C S$. Pancreases, liver, spleen, brown adipose tissue and thymus have higher levels of Fads3AT9 compared to Fads3CS. Data is expressed as mean $\pm \mathrm{SD}(\mathrm{n}=4)$ and asterisk represents $P<0.05$ 


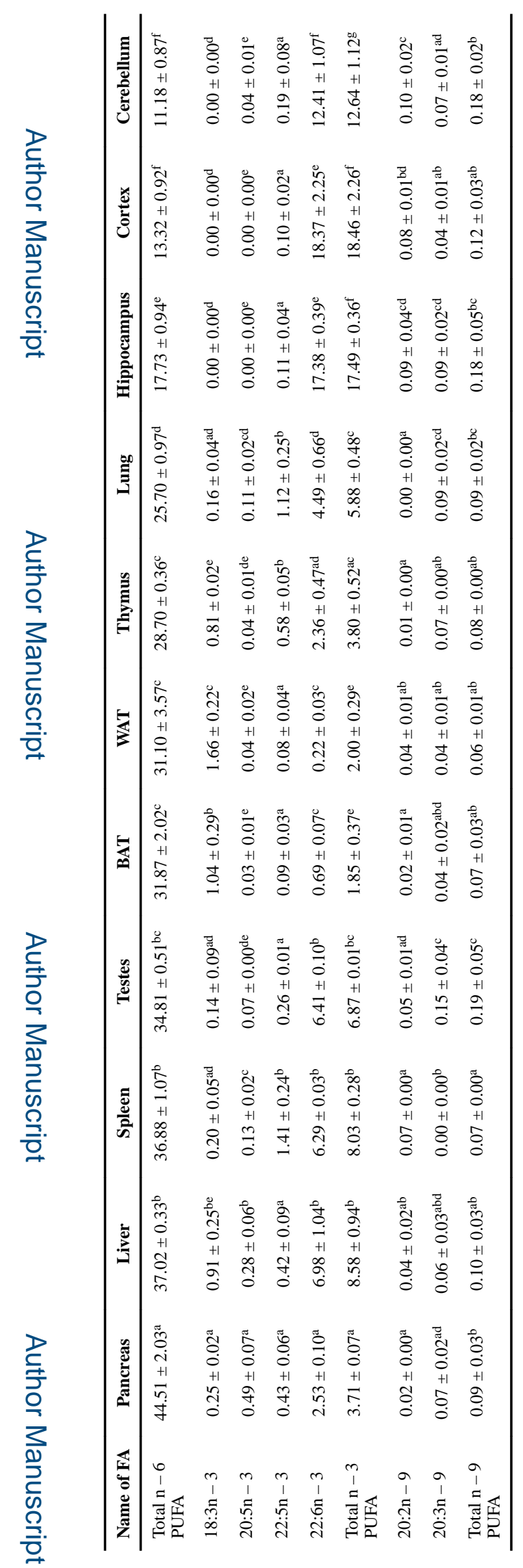

Mol Biol Rep. Author manuscript; available in PMC 2017 August 01. 